\title{
Risk Assessment Model of Residual Coal Spontaneous Combustion Based on Combination Weighting and SPA Method and Its Application
}

Yun Qi ( $\nabla$ qiyun_sx@sxdtdx.edu.cn )

Shanxi Datong University

\section{Wei Wang}

Shanxi Datong University

Jiaqi Ge

Tashan Coal Mine, Jinneng Holding Coal Industry Group

\section{Zebin Yang}

Changzhi Power Supply Company of State Grid Shanxi Electric Power Company

\section{Qingjie Qi}

EmergencyResearch Institute

\section{Research Article}

Keywords: prediction of spontaneous combustion risk, comprehensive weight, compromise coefficient, improved combination weight-SPA evaluation model, maximum membership

Posted Date: March 7th, 2022

DOI: https://doi.org/10.21203/rs.3.rs-1409821/v1

License: (9) This work is licensed under a Creative Commons Attribution 4.0 International License.

Read Full License 


\title{
Risk Assessment Model of Residual Coal Spontaneous Combustion Based on Combination Weighting and SPA Method and Its Application
}

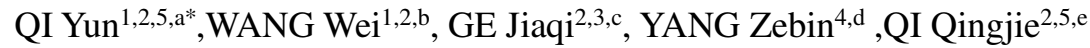 \\ 1 School of Coal Engineering,Shanxi Datong University,Datong Shanxi 037000,China;
}

2 College of Safety Science and Engineering, Liaoning Technical University,Fuxin Liaoning 123000,China;

3 Tashan Coal Mine, Jinneng Holding Coal Industry Group, Datong Shanxi 037000,China;

4 Changzhi Power Supply Company of State Grid Shanxi Electric Power Company, Changzhi Shanxi 046000, China.

5 China Coal Technology Engineering Group, EmergencyResearch Institute, BeiJing, 100013,China;

\begin{abstract}
In order to solve the problem of low accuracy about the autogenous risk assessment in minded-out area, which caused several kinds of risk factors like ambiguity, arbitrament, uncertainty, variability and diversity, a combination weighting set pair analysis (SPA) coupling evaluation should be put forward. Firstly, based on the mechanism and occurrence conditions of coal spontaneous combustion, There are 11 influencing factors identified and extracted from the three aspects, which are named as coal spontaneous combustion tendency, extraction conditions, air leakage and heat storage conditions; Then, euclidean measure should be introduced to make sure the degree in difference between the subjective and objective weights and compromising modulus, so the combined weights of each index were obtained. Based on SPA theory, a combined weighting spa coupling mining model was constructed; Finally, Setting three minded-out areas affected by gas drainage conditions in Ping Dingshan No.10 Coal Mine as an example, the set pair association degree expression of evaluation index and corresponding standard is obtained, and finally the risk level was determined. The results show that the prediction results of spontaneous combustion risk in No.1, No.2 and No.3 minded-out areas are consistent with the measured results, and the prediction accuracy of the model is high.
\end{abstract}

Keywords: prediction of spontaneous combustion risk; comprehensive weight; compromise coefficient; improved combination weight-SPA evaluation model; maximum membership

\footnotetext{
* a Yun QI(1988-) Male, Huaibei City, Anhui Province, China, PhD, lecturer. Main research direction is mine fire prevention and control, coal mine gas disaster prevention and control theory and technology, Email: qiyun_sx@sxdtdx.edu.cn

b Wei WANG(1991-), male, Tangshan City, Heibei Province, PhD, Lecturer. His main research direction is mine fire prevention and control, coal mine gas disaster prevention, control theory, and technology, Email: wangwei@sxdtdx.edu.cn

c Jiaqi GE(1994-), memale, Datong city,Shanxi Province,China, Master, Main research directions: Mine disaster prevention, theory and technology of mine fire prevention and control.E-mail: g325815@163.com

d Zebin YANG(1990-),Male, Changzhi city,Shanxi Province,China, Master, Main research directions : Sliding electrical contact theory and application.E-mail: czsyangzebin@163.com

e Qingjie QI(1964-),male,Chaoyang City,Liaoning Province, PhD, professor, Main research directions: theory and technology of mine fire prevention and control, Email: DTDX513523736@163.com.

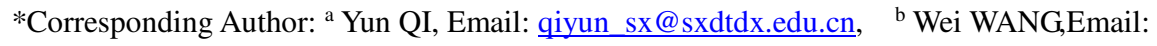

wangwei@sxdtdx.edu.cn
} 


\section{Introduction}

Spontaneous combustion of residual coal in minded-out area is one of the main factors threatening coal mine safety production (Zhan et al.2017; Zhan et al.2020). With the continuous increasing of coal mining depth, the original rock temperature, ground pressure and gas content, the risk of spontaneous combustion in minded-out area increased, especially under the influence of gas drainage. When the air leakage intensity in goaf is large, the risk of spontaneous combustion of residual coal in goaf is more prominent (Wang et al.2014). Therefore, it is of great significance to study the risk prediction of spontaneous combustion in goaf affected by gas drainage.

Spontaneous combustion of residual coal in goaf is a latent and uncertain complex physical and chemical process, and its inducement often has a strong nonlinear effect (Luo.2017). Once the fire occurs, the risk expansion speed is very fast, because the ignition location is relatively hidden, so it is difficult to accurately determine the ignition status in goaf. (Sun et al.2017). Therefore, scholars both at home and abroad have done a lot of research towards the risk prediction methods of coal spontaneous combustion from different angles. For example:Sahu et al(Sahu et al.2011)proposed the fuzzy c-means method based on the principle of fuzzy expert system and artificial neural network, and have applied it to the prediction of coal spontaneous combustion tendency to verify the accuracy of the model. Zhao Linlin et al(Zhao Linlin et al.2018) used PCA AdaBoost model to predict the risk of coal spontaneous combustion in goaf under unbalanced data to improve the accuracy of prediction. The results show that the prediction result is obviously better and more effective than PSO-SVM model. Xiao Hongfei et al(Xiao Hongfei et al.2001) applied adaptive step method and annealing algorithm to improve the evaluation model of BP neural network, and made a great contribution to improve the accuracy of spontaneous combustion risk prediction of mining seam. Wang Wei et al(Wang Wei et al.2019) established the G2-TOPSIS decision-making model of borehole spontaneous combustion risk based on the approximate ideal solution and modified G2 weighting method by introducing difference driving method. Chen Jinquan et al(Chen Jinquan et al.2019) based on the theory of partial order set, used the partial order set evaluation model to construct the hazard Hasse diagram of spontaneous combustion in goaf, and analyzed the hazard degree of spontaneous combustion in goaf. Li Tongsuo et al( $\mathrm{Li}$ Tongsuo et al.2013) established set pair analysis model to predict the risk of spontaneous combustion of residual coal in goaf. However, the above prediction models have their own merits and drawbacks. For example, although BP neural network method can avoid the subjective influence of artificially determining the weight coefficient, the difficulty of obtaining the sample information still affect the quality of the solution; Additionally, Even if SPA method can make full use of the interaction between certainty and uncertainty, but the risk level classification is easily unclear and the evaluation result is distorted in the evaluation process, and etc.

In view of this, the author intends to combine the improved Analytic Hierarchy Process (AHP) method with Entropy Weight Method(EWM). Meanwhile, the spontaneous combustion of residual coal in goaf is regarded as a system which can have mutual influence between certainty and uncertainty. According to the extensibility of SPA theory, the traditional set pair analysis method is optimized, and the risk evaluation model of spontaneous combustion in goaf is established based on the combination weighting SPA coupling. Taking the goaf of F group coal seam in No.10 mine of Pingdingshan Coal Mine as the research background, the gas drainage effect is mainly 
considered In order to lay the foundation for taking scientific and reasonable measures to prevent spontaneous combustion in goaf, the influence of gas drainage on spontaneous combustion in goaf is analyzed, and the risk of spontaneous combustion of residual coal in goaf is predicted.

\section{Determination of combination weight}

\subsection{Combination weighting principle}

In order to avoid the fact that the decision-maker's subjective will influence on the risk of spontaneous combustion in goaf, which can not reflect the actual data, and the objective survey data is easily affected by discrete extreme value, and two or more methods to determine the weight should be combined, so their respective advantages are used to obtain the combined weight (Lin Haifei,et al.2020). In this paper, euclidean measure is introduced to couple the subjective weight determined by AHP method with the objective weight determined by EWM method, so as to ensure that the difference between the subjective weight and the objective weight, which is consistent with the corresponding compromise coefficient. Finally, the calculation method of "AHP + EWM" coupling is formed to determine the ideal weight of goaf spontaneous combustion risk assessment index.

\subsection{Determination of subjective weight by AHP}

AHP is a decision-making method which can comprehensively analyze complex multi-objective decision-making problems by combining qualitative and quantitative analysis [Chen Liuyu et al.2020]. The basic idea is listed as follows: firstly, analyzing the system decision-making objectives and establishing a multi factor hierarchical structure model describing the system functions or characteristics. Then, the relative importance of each factor is determined by using the comparative scaling method to construct the judgment matrix of the relative importance of each index. Finally, the maximum eigenvalue and its corresponding eigenvector are calculated, and the consistency of the importance judgment matrix is verified. The feature vector is the weight vector of evaluation index after standardization. The main steps of using AHP to calculate index weight are as follows:

1)The hierarchical structure model of spontaneous combustion risk assessment system is established. Firstly, the relationship and influence on the basic elements in the goaf spontaneous combustion risk assessment system should be analyzed. Additionally, expert opinions should also be combined to divide the indexes into index layer, criterion layer and target layer.

2) The judgment matrix is constructed. According to the comparative scaling method, the importance of each index factor relative to a certain criterion of the upper layer should be compared and analyzed, so the judgment matrix is constructed $\boldsymbol{A}=\left(a_{i j}\right)_{m \times n}$ :

$$
\boldsymbol{A}=\left[\begin{array}{cccc}
a_{11} & a_{12} & \mathrm{~L} & a_{1 n} \\
a_{21} & & \mathrm{~L} & a_{2 n} \\
\mathrm{M} & \mathrm{M} & \mathrm{O} & \mathrm{M} \\
a_{m 1} & a_{m 2} & \mathrm{~L} & a_{m n}
\end{array}\right]
$$

3) The index factors are ranked in hierarchy. The square root method is used to calculate the weight, which corresponds to the relative importance of each index factor in the judgment matrix 
under the criterion, and the obtained vector $\beta=\left(\beta_{1}, \beta_{2}, \cdots, \beta_{n}\right)$ is normalized to obtain the eigenvector corresponding $w$ to the maximum eigenvalue $\lambda_{\max }$ of the judgment matrix $\boldsymbol{A}$, that is, the relative importance weight of each index factor in the same layer to a certain index in the upper layer

$$
\begin{gathered}
\beta_{i}=\sqrt[n]{\begin{array}{c}
n \\
a_{i j} \\
j=1
\end{array}}, i=1,2,3, \ldots, n \\
w_{i}=\frac{\beta_{i}}{\sum_{j=1}^{n} \lambda_{i}}, i=1,2,3, \ldots, n
\end{gathered}
$$

So we get $w=\left(w_{1}, w_{2}, \ldots, w_{n}\right)^{T}$, which is the approximate value of the normalized eigenvector where $w_{i}$ is the weight corresponding to the $i^{\text {th }}$ factor and $\sum_{i=1}^{n} w_{i}=1$.

4) Consistency test of judgment matrix. Generally, it is difficult to judge whether the judgment matrix meets the requirements of complete consistency in the construction process or not. Therefore, a series of measurement standard are necessarily established. When the judgment matrix meets this standard, it is approximately considered that it has complete consistency, and the weight of each level index, which is calculated by the judgment matrix with complete consistency, is reasonable.

$$
\begin{aligned}
\lambda_{\max } & =\frac{1}{n} \frac{(A \cdot w)_{i}}{w_{i}} \\
C R & =\frac{C I}{I} R \\
C I & =\frac{\left|\lambda_{\text {max }}-n\right|}{\delta-1}
\end{aligned}
$$

In the formula, $C R$ is the consistency ratio; $C I$ is the consistency index; $R I$ is the random consistency index, which can be determined by looking up the chart [Luo Dayong.2017]. Only when $C R<0.1$ is established, can the judgment matrix meet the requirement of consistency. Otherwise, it needs to be rebuilt until the requirement of consistency is provided.

\subsection{Determination of subjective weight by EWM method}

EWM is an objective weighting method which uses the effective information contained in the measured data of various factors affecting the decision-making objectives to judge the degree of dispersion. When the variation degree of the evaluation index of spontaneous combustion risk in goaf is greater and the entropy value is smaller, the fact is that the amount of effective information provided by the evaluation index and the weight in the comprehensive evaluation of spontaneous combustion risk will be both greater. The weight calculation steps of EWM method are as follows:

1)The decision matrix $\boldsymbol{R}$ is constructed. Based on the principles of the combination of qulalitive and quantatitative analysis, the $m$ samples can be nondimensionalized and standardized, which are related with the risk of assessment of spontaneous combustion, so the original data matrix of $n$ spontaneous combustion risk evaluation indicators can be obtained 


$$
r_{i j}=\left\{\begin{array}{l}
\frac{b_{i j}-\min b_{j}}{\max b_{j}-\min b_{j}}, \text { income-type index } \\
\frac{\max b_{j}-b_{i j}}{\max b_{j}-\min b_{j}}, \text { cost-type index }
\end{array}\right.
$$

In the formula, $\min b_{j}$ and $\max b_{j}$ are the best and worst values of each evaluation factor relative to a certain index.

2) The entropy value of evaluation index is determined. In order to avoid the fact that the index characteristic proportion value is zero, ensuring that the evaluation index entropy has mathematical significance, controlling the influence of $r_{i j} \cdot \ln r_{i j}$ on the index entropy within a reasonable range, so it is necessary to modify some factors in the decision matrix, and then determine the evaluation index entropy value:

$$
\begin{gathered}
x_{i j}=\left\{\begin{array}{c}
\frac{r_{i j}}{\sum_{i=1}^{n} r_{i j}}, r_{i j} \neq 0 \\
\frac{r_{i j}+\psi}{\sum_{i=1}^{n}\left(r_{i j}+\psi\right)}, r_{i j}=0
\end{array}\right. \\
e_{j}=-\sum_{i=1}^{m} r_{i j} \cdot \ln r_{i j}
\end{gathered}
$$

In the formula, $x_{i j}$ is the characteristic proportion of rating indicators, $\psi$ is the correction factor, $e_{j}$ is the entropy weight of the $j^{\text {th }}$ index .

3) Calculate the information weight of index $i$ :

$$
w_{j}=\frac{1-e_{j}}{\sum_{j=1}^{n}\left(1-e_{j}\right)}, \text { Where } \sum w_{j}=1
$$

\subsection{Using "AHP + EWM" method to determine combination weight}

To ensure that the decision-makers' subjective understanding of the risk factors of spontaneous combustion in goaf and the objective investigation data can truly reflect the law, the compromise coefficient is introduced to couple the subjective and objective weights of each index factor; and the Euclidean measure in n-dimensional space is introduced to ensure the consistency of the difference between the subjective and objective weights and the compromise coefficient:

$$
\begin{gathered}
w=\alpha w_{\mathrm{A}}+(1-\alpha) w_{\mathrm{E}} \\
D\left(w_{A j}, w_{B j}\right)=\sum_{j}^{n}\left(w_{A j}-w_{B j}\right)^{2} \\
D\left(w_{A j}, w_{B j}\right)^{2}=(\alpha-1)^{2}
\end{gathered}
$$

In the formula, $\alpha$ is the weight compromise coefficient, $D\left(w_{A j}, w_{E j}\right)$ is an Euclidean metric, 
$D\left(w_{A j}, w_{E j}\right)^{2}$ is the degree of difference between subjective and objective weight and compromise coefficient.

\section{Evaluation model construction of combination weighting and spa coupling}

\subsection{Construction of set pair analysis model}

With the requirements of safety evaluation accuracy in engineering practice, the identical discrepancy contrast connection degree model in the traditional set pair analysis theory is too rough and simple to distinguish the grade difference when the evaluation index is in the adjacent or interval (Lin Yongliang et al,2019). Therefore, it is necessary to optimize the expansibility of traditional connection degree.

1) Suppose that the set of multifactor evaluation is $U=\left\{u_{1}, u_{2}, \ldots, u_{n}\right\}$, and many experts invited to grade the evaluation index of the evaluation factor $u_{s}(1 \leqslant s \leqslant n)$ to form the grade vector $V=\left\{v_{1}, v_{2}, \ldots, v_{m}\right\}$, Additionally, the evaluation target towards identical discrepancy contrast evaluation of the evaluation factor $u_{s}$ is $r_{s 1}+r_{s 2} i+\mathrm{L}+r_{s m-1} i+r_{s m} j$, where $r_{s 1}+r_{s 2}+\mathrm{L}+r_{s m-1}+r_{s m}=1$, and then the initial identical, dissimilar and inverse evaluation matrix $\boldsymbol{R}$ is obtained:

$$
\boldsymbol{R}=\left(\begin{array}{cccc}
r_{11} & r_{12} & \mathrm{~L} & r_{1 m} \\
r_{21} & r_{22} & \mathrm{~L} & r_{2 m} \\
\mathrm{M} & \mathrm{M} & \mathrm{O} & \mathrm{M} \\
r_{n 1} & r_{n 2} & \mathrm{~L} & r_{n m}
\end{array}\right)
$$

In the formula, $i$ is the difference coefficient, $i \in[-1,1], j$ is the coefficient of opposition, $j=-1$.

2) the matrix of multiple coefficients $\boldsymbol{E}=\left(\begin{array}{llllll}1 & i_{1} & i_{2} & \mathrm{~L} & i_{m-2} & j\end{array}\right)^{T}$ constituted by evaluating the set or set pair on the coefficients of degree of identity, degree of difference and degree of opposition of the evaluation set or set pair under certain background conditions, and the weight vector $W=\left(\begin{array}{llll}w_{1} & w_{2} & \mathrm{~L} & w_{n}\end{array}\right)$ of each index factor in the evaluation set $U$ combine together, so the same, different and opposite evaluation model is constructed:

$$
\mu=\boldsymbol{W} \cdot \boldsymbol{R} \cdot \boldsymbol{E}=\left(\begin{array}{c}
w_{1} \\
w_{2} \\
\mathrm{M} \\
w_{n}
\end{array}\right)^{T} \cdot\left(\begin{array}{cccc}
r_{11} & r_{12} & \mathrm{~L} & r_{1 m} \\
r_{21} & r_{22} & \mathrm{~L} & r_{2 m} \\
\mathrm{M} & \mathrm{M} & \mathrm{O} & \mathrm{M} \\
r_{n 1} & r_{n 2} & \mathrm{~L} & r_{n m}
\end{array}\right) \cdot\left(\begin{array}{c}
1 \\
i_{1} \\
\mathrm{M} \\
i_{m-2} \\
j
\end{array}\right)
$$

Namely :

$$
\mu=\sum_{s=1}^{n} w_{s} r_{s 1}+\sum_{s=1}^{n} w_{s} r_{s 2} i_{1}+\sum_{s=1}^{n} w_{s} r_{s} i_{2}+\mathrm{L}+\sum_{s=1}^{n} w_{s} r_{s l} i_{m-1}+\sum_{s=1}^{n} w_{s} r_{s m} j
$$




\subsection{Constructing the evaluation model of combination weighting SPA coupling}

The connection degree $u_{s k}$ is obtained by assuming the connection degree $u_{s}$ as the $s^{\text {th }}$ evaluation object and analysing the set pair analysis of the $k^{\text {th }}$ index in the evaluation index. Combined with the calculation of the above combination weight, a SPA model is constructed

$$
\mu_{s}=w_{k} u_{s k}
$$

Combining with the formula (11), (15) and (16), we can get the following:

$$
\mu=\sum_{s=1}^{n} w r_{s 1}+\sum_{s=1}^{n} w r_{s 2} i_{1}+\sum_{s=1}^{n} w r_{s 1} i_{2}+\mathrm{L}+\sum_{s=1}^{n} w r_{s 1} i_{m-1}+\sum_{s=1}^{n} w r_{s m} j
$$

Combination weighting - SPA coupling evaluation model, making full use of SPA theory and considering the uncertainty characteristics of evaluation indexes, and combined weight introduced to quantify the evaluation indexes are to optimize the evaluation model of goaf spontaneous combustion and improve the accuracy of evaluation results. The research framework is shown in Figure 1.

\section{Application of prediction example of spontaneous combustion in goaf}

\subsection{Evaluation index of spontaneous combustion grade in goaf}

Goaf spontaneous combustion is the result of multiple factors. It is generally believed that spontaneous combustion is only caused by accumulating enough heat under the condition of continuous air leakage and oxygen supply in the goal residual coal. Affected by the extraction conditions, the conditions of air leakage and oxygen supply in goaf and heat storage in coal seam change dynamically with strong uncertainty. According to the actual situation of the coal seams in No.10 Coal Mine of Pingdingshan Coal Mine, the evaluation index system of spontaneous combustion risk of goaf with 11 single indexes is established with the spontaneous combustion tendency of coal, extraction conditions, air leakage and heat storage conditions as the main factors, as shown in Table 1, considering the factors affecting the spontaneous combustion risk of goaf comprehensively.

In reference [Feng zhibin,2015], the risk degree of spontaneous combustion in goaf is divided into five levels: I (safe), II (relatively safe), III (generally safe), IV (relatively unsafe) and V (unsafe), as shown in Table 2. The identical discrepancy contrast evaluation of evaluation object relative to evaluation factor $u_{s}$ is $r_{s 1}+r_{s 2} i+r_{s 3} i+r_{s 4} i+r_{s 5} j$, in which $r_{s 1}+r_{s 2}+r_{s 3}+r_{s 4}+r_{s 5}=1$. Based on the principle of average distribution, $i_{1}=0.5, \quad i_{2}=0, i_{3}=0.5$ and $j=-1$ are taken into the expression of connection degree, and the corresponding risk level can be obtained by calculating the value of connection degree.

\subsection{Determination of comprehensive weight of " AHP+EWM "}

According to the comparison method towards the important degree of Group F goaf of Pingdingshan No.10 Coal Mine, $1 \sim 9$ scale method is used to quantitatively construct the judgment matrix. According to formula (1) (3), the eigenvector corresponding to $\lambda_{\max }$ is obtained : $W=(0.3333,0.3333,0.3333) \quad, \quad W_{1}=(0.3873,0.1698,0.4429) \quad, \quad W_{2}=(0.4905,0.3119,0.1976)$, 
$W_{3}=(0.3362,0.2656,0.2212,0.0885,0.0885)$. By using formula $(4) \sim(6)$ to test the consistency of eigenvalues, it can be seen that $C R$ values of each judgment matrix meet $C R<0.1$, Therefore, if the judgment matrix and eigenvector meet the requirements, the weight vector corresponding to "AHP" method is as follows: $w_{A 1}=(0.3873,0.1698,0.4429), w_{A 2}=(0.4905,0.3119,0.1976)$

$w_{A 3}=(0.3362,0.2656,0.2212,0.0885,0.0885)$. Then, according to the data in Table 1 , the original data of the evaluation object are treated with dimensionless normalization, and the weight corresponding to the "EWM" method of goaf spontaneous combustion risk factor is obtained through formula $(8) \sim(10): w_{E 1}=(0.3021,0.3413,0.3566) \quad, \quad w_{E 2}=(0.2968,0.3294,0.3738)$, $w_{E 3}=(0.1796,0.1666,0.2008,0.2303,0.2227)$. Euclidean measure is introduced to combine the subjective weight of AHP method with the objective weight of EWM method. According to the formula (11) to (13), the compromise coefficient $\alpha$ is 0.73 . Finally, the ideal comprehensive weight of each evaluation index is obtained: $w_{1}=(0.3643,0.2161,0.4196), w_{2}=(0.4382,0.3166,0.2452)$,

$w_{3}=(0.2939,0.2389,0.2157,0.1268,0.1247)$. From the comparison of comprehensive weight, it can be seen that the different evaluation indexes have different effects on the spontaneous combustion risk of goaf. The primary and secondary factors affecting the spontaneous combustion risk of goaf are $U_{21}>U_{13}>U_{11}>U_{22}>U_{31}$, which can be used as the main criterion for the prevention and control of spontaneous combustion risk of goaf.

\subsection{Determination of risk grade of spontaneous combustion in goaf}

According to the evaluation index system and the goaf data of Pingdingshan No.10 Coal Mine collected in literature(Zhang Jianguo,et al. 2019), experts with solid theoretical knowledge and rich practical experience are invited to score the three goaf evaluation index factors to be predicted, and the evaluation results are normalized, as shown in Table 3.

According to table 3 , the initial same, different, and reverse evaluation matrix is obtained. Combined with formulas (15) and (17), the connection degree expression of evaluation result of No.1 goaf is constructed as follows: 


$$
\begin{aligned}
& \mu_{11}=\left(w_{1}, w_{2}, \mathrm{~L}, w_{n}\right) \cdot\left(\begin{array}{ccccc}
r_{11} & r_{12} & r_{13} & r_{14} & r_{15} \\
r_{21} & r_{22} & r_{23} & r_{24} & r_{15} \\
\mathrm{M} & \mathrm{M} & \mathrm{M} & \mathrm{M} & \mathrm{M} \\
r_{n 1} & r_{n 2} & r_{n 3} & r_{n 4} & r_{n 5}
\end{array}\right) \cdot\left(\begin{array}{c}
1 \\
i_{1} \\
i_{2} \\
i_{3} \\
j
\end{array}\right) \\
& =(0.3643,2161,0.4196) \cdot\left(\begin{array}{ccccc}
0.07 & 0.45 & 0.28 & 0.16 & 0.04 \\
0.05 & 0.39 & 0.36 & 0.16 & 0.04 \\
0.07 & 0.42 & 0.3 & 0.14 & 0.07
\end{array}\right) \cdot\left(\begin{array}{c}
1 \\
i_{1} \\
i_{2} \\
i_{3} \\
j
\end{array}\right) \\
& =0.0657+0.4244 i_{1}+0.3057 i_{2}+0.1515 i_{3}+0.0526 j
\end{aligned}
$$

In the same way:

$$
\begin{aligned}
& \mu_{12}=0.0772+0.4204 i_{1}+0.3315 i_{2}+0.12 i_{3}+0.0509 j \\
& \mu_{13}=0.2032+0.3908 i_{1}+0.2593 i_{2}+0.1091 i_{3}+0.0376 j
\end{aligned}
$$

Similarly, the relation degree expression of No.2 goaf evaluation result is as follows:

$$
\begin{aligned}
& \mu_{21}=0.0775+0.3384 i_{1}+0.4220 i_{2}+0.0980 i_{3}+0.06420 j \\
& \mu_{22}=0.0787+0.3193 i_{1}+0.4076 i_{2}+0.1281 i_{3}+0.0663 j \\
& \mu_{23}=0.2282+0.2514 i_{1}+0.3754 i_{2}+0.1002 i_{3}+0.0448 j
\end{aligned}
$$

The relation degree expression of No.3 goaf evaluation result is as follows:

$$
\begin{aligned}
& \mu_{31}=0.2486+0.4490 i_{1}+0.1706 i_{2}+0.0897 i_{3}+0.0421 j \\
& \mu_{32}=0.2405+0.4678 i_{1}+0.1475 i_{2}+0.0968 i_{3}+0.0474 j \\
& \mu_{33}=0.3619+0.3032 i_{1}+0.2172 i_{2}+0.0604 i_{3}+0.0573 j
\end{aligned}
$$

By calculating the evaluation results of each goaf, the average connection degree values of No.1 and No.3 goaf are 0.2138 and 0.3712 respectively, and their evaluation grades are grade II (relatively safe); The average connection degree of No.2 goaf is 0.1708 , and its evaluation grade is grade III (general safety). The evaluation results of spontaneous combustion risk in goaf are shown in Table 4. The results are consistent with the actual situation of goaf spontaneous combustion risk, and the accuracy of the evaluation model is close to $100 \%$. Therefore, the evaluation model has certain universality and guiding significance.

\section{Conclusion}

1) The combination weighting method is used to ensure the reasonable distribution of the weight of each index factor of goaf spontaneous combustion risk prediction, but analyzing the weight value of each index shows that there are some difference towards the degree of influence 
of goaf spontaneous combustion risk factors on spontaneous combustion risk, and the main factors affecting the spontaneous combustion risk of goaf in Pingdingshan No.10 coal mine are $U_{21}>U_{13}$ $>U_{11}>U_{22}>U_{31}$.

2) The evaluation from the combination weighting SPA coupling model for No.1, No.2 and No.3 goaf spontaneous combustion risk prediction results are basically consistent with the measured results, only No.2 goaf evaluation results are relatively conservative. But from the risk point of view, the evaluation results are more reliable, which may be consistent with the phenomenon that the measured results of coal spontaneous combustion marker gas concentration and temperature growth rate of some gas drainage boreholes in the mine are higher.

3) When predicting the spontaneous combustion risk of Pingdingshan No.10 coal mine by using the combined weighting spa coupling goaf spontaneous combustion risk evaluation model, not only can the calculation results be clearly and effectively to reflect the level of each evaluation index, but also sort the risk level according to the connection degree parameters of each index, so that the evaluation results have higher accuracy, which provides the foundation for reasonable prevention and control of spontaneous combustion in goaf.

\section{Abbreviations}

AHP: Analytic Hierarchy Process

EWM: Entropy Weight Method

SPA: Set Pair Analysis

\section{Declarations}

\section{Acknowledgments}

The research presented in this paper was supported by the Key Laboratory of Mine Thermodynamic disasters and Control of Ministry of Education (Liaoning Technical University), Huludao, China. We thank LetPub (www.letpub.com) for its linguistic assistance during the preparation of this manuscript.

\section{Funding}

The National Natural Science Foundation of China (Grant: 51274113);

Key special-funded projects of the State key R \& D program (2018YFC0807900);

Doctoral research startup project of Shanxi Datong university (2020-B-18,2020-B-08)

\section{Availability of data and materials}

Applicable.

\section{Competing interests}


The authors declare that they have no competing interests.

\section{Authors' Affiliations}

1. School of Coal Engineering, Shanxi Datong University, Datong Shanxi 037000, China;

2. College of Safety Science and Engineering, Liaoning Technical University, Fuxin Liaoning 123000, China;

3.Tashan Coal Mine, Jinneng Holding Coal Industry Group, Datong Shanxi 037000,China;

4. Changzhi Power Supply Company of State Grid Shanxi Electric Power Company, Changzhi Shanxi 046000, China.

5. China Coal Technology Engineering Group, Emergency Research Institute, Beijing, 100013, China;

\section{Reference}

[1]ZHAN Qi, WANG Xuefeng, HUANG Ge, et al. Study on goaf gas drainage technology and float coal spontaneous combustion coupling control[J].Coal Science and Technology, 2017,45(10):111-116.

[2]ZHANG Jufeng, SHI Shiliang, LU Yi, et al. Symbiotic disasters of mine gas and coal spontaneous combustion:coupling relationship, disaster mechanism, prevention and control technology [J]. China Safety Science Journal,2020,30(10):149-155.

[3]WANG Mingchong, LIU Zegong, ZHANG Xiaojian, et al. Research of risk assessment on spontaneous combustion of goaf coal based on Analytic Hierarchy Process and extended set pair theory[J].Journal of Safety Science and Technology,2014,10(8):182-188.

[4]LUO Dayong. Cause analysis of spontaneous combustion of coal in gob[J]. Mining Safety \& Environment Protection,2017,44(5),107-110.

[5]SUN Zheng, WEN Changping, BAI Yinyong, et al. Evaluation of goaf coal spontaneous combustion based on attribute mathematical theory[J]. Journal of PLA University of Science and Technology,2017,(4):1-5.

[6]SAHU H B, PADHEE S, MAHAPATRA S S. Prediction of spontaneous heating susceptibility of indian coals using fuzzy logic and artificial neural network models[J]. Expert Systems with Applications,2011,38(3):2271-2282.

[7]ZHAO Linlin,WEN Guofeng,SHAO Liangshan. PCA-AdaBoost model for predicting coal spontaneous combustion in caving zone with imbalanced data[J]. China Safety Science Journal,2018,28(3):74-78.

[7]HUANG Mengtao,ZHAO Yiqun, LIU Ying. Application of neural network to modelling of coal spontaneous combustion stove at low temperature[J]. Journal of Xian Mining Institute,1996,16(1):76-79.

[8]XIAO Hongfei, LIU Liming, WANG Haiqiao. The application of modified BP neural network to predict mine coal layer spontaneous combustion[J]. Journal of China Coal Society, 2001,26(6):649-653.

[9]WANG Wei,JIA Baoshan,QI Yun. Prediction model of spontaneous combustion risk of extraction drilling based on improved CRITIC modified G2-TOPSIS method and its application[J]. China Safety Science Journal, 2019,29(11):26-31.

[10]CHEN Jinquan, WANG Jiren, YUE Lizhu. Evaluation model on possibility of coal spontaneous combustion in goaf based on partially ordered set[J].Journal of Safety Science and Technology ,2019,15(2):89-93. 
[11]LI Tongsuo, HE Qilin. Application of set pair analysis in the risk evaluation of coal spontaneous combustion[J]. Journal of Safety Science and Technology, 2013,9(8):54-58.

[12]LIN Haifei,GAO Fan,YAN Min. Study on PSO-BP neural network prediction method of coal seam gas content and its application[J]. China Safety Science Journal, 2020,30(9):80-87.

[13]CHEN Liuyu,LI Xijian,BI Juan,et al. Prediction of coal-gas outburst induced by rock-burst tendency based on AHP-TOPSIS

[14]LIN Yongliang, ZHAO Lei. Monitoring and analysis for goaf spontaneous combustion during gas extraction[J]. Safety in coal mines, 2019,15(4):1239-1245.

[15] Feng Zhibin.Research on fire risk assessment of construction site based on set pair analysis[D].Xi'an: Xi'an University of Architecture and Technology,2015.

[16]Zhang Jianguo,Qi Yingjie,Lyu Youchang et al. Research on integrated technology of high efficient gas drainage in deep mine [R].Fuxin:辽 Liaoning Technology University,2019.

\section{First author:}

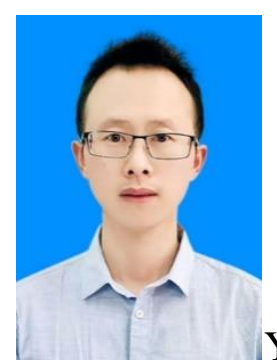

Yun QI(1988-) Male, Huaibei City, Anhui Province, China, PhD, lecturer. Main research direction is mine fire prevention and control, coal mine gas disaster prevention and control theory and technology, E-mail:qiyun_sx@sxdtdx.edu.cn

\section{Corresponding Author:}

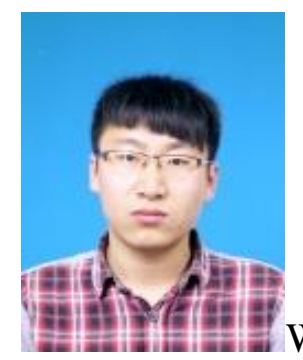

Wei WANG(1991-), male, Tangshan City, Heibei Province, PhD, Lecturer. The main research direction is mine fire prevention and control, coal mine gas disaster prevention, control theory, and technology. Email:wangwei@sxdtdx.edu.cn

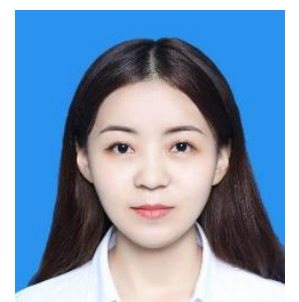

Jiaqi GE (1994-),Female, Datong city,Shanxi Province,China, Master, 
Main research directions: Mine disaster prevention, theory and technology of mine fire prevention and control.E-mail: g325815@163.com

\section{Other authors:}

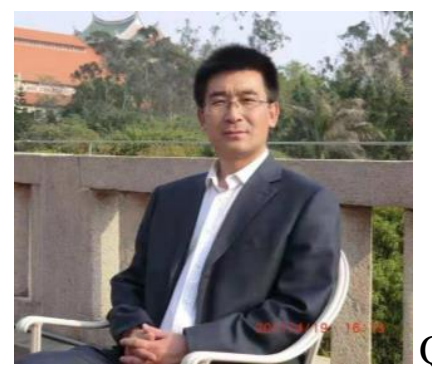

Qingjie QI(1964-),male,Chaoyang City,Liaoning Province, $\mathrm{PhD}$, professor, Main research directions: theory and technology of mine fire prevention and control. Email: DTDX513523736@163.com

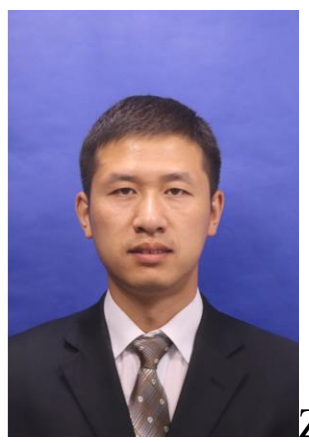

Zebin YANG(1990-),Male, Changzhi city,Shanxi Province,China, Master, Main research directions : Sliding electrical contact theory and application.E-mail: czsyangzebin@ 163.com 


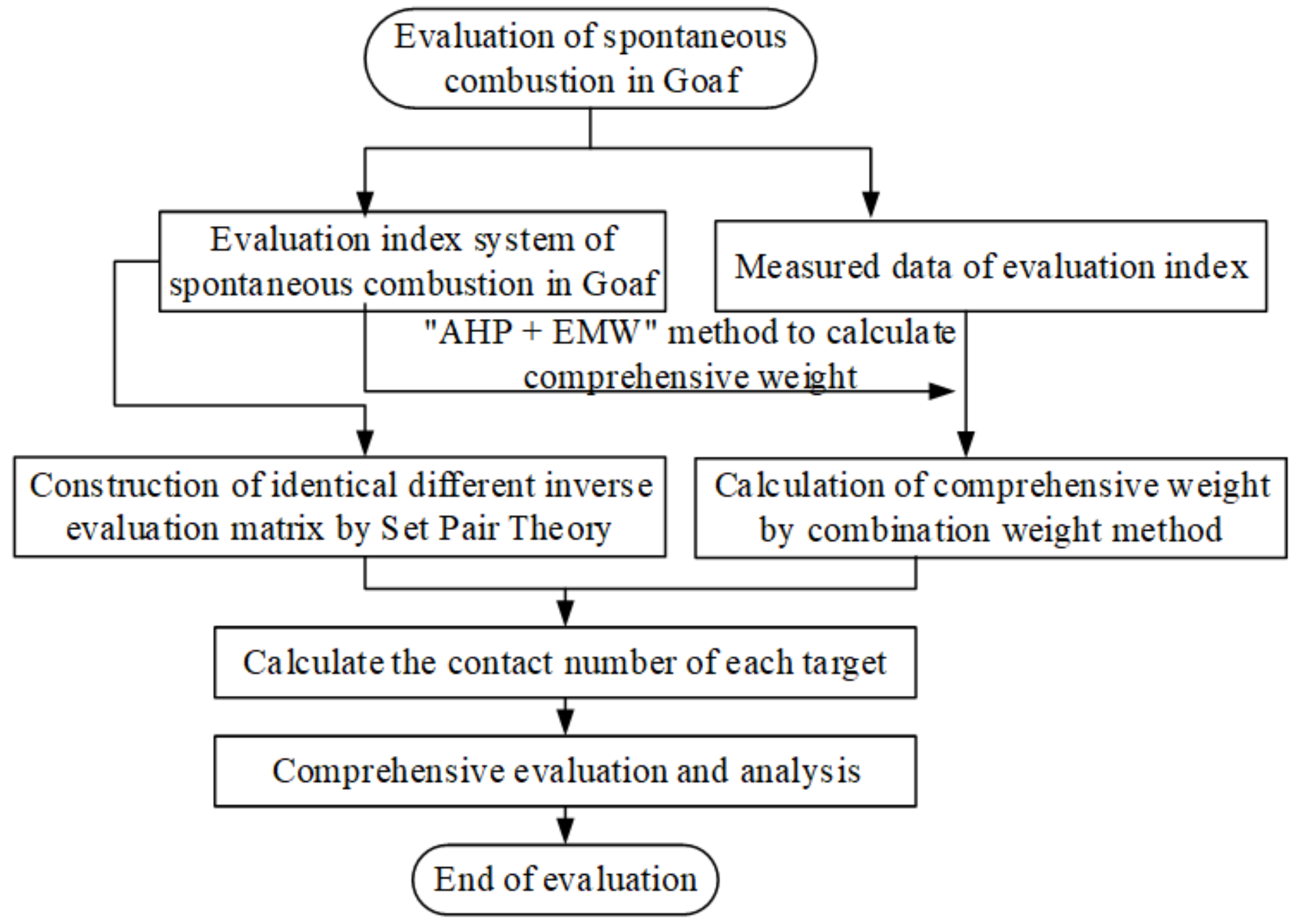

Figure 1

Improve the SPA evaluation process of portfolio weight 